\title{
VERY HIGH VOLTAGE PHOTOEMISSION ELECTRON GUNS*
}

\begin{abstract}
There are a growing number of applications for $\mathrm{CW}$ electron accelerators, many requiring high average current and small transverse and longitudinal emittances. Very high voltage DC electron guns with photoemission cathodes are a natural choice for generating the beams for these machines. High average current applications require high quantum efficiency photocathodes at practical wavelengths. The necessary lasers are state-of-the-art systems that must be considered in choosing a photocathode. Field emission from the electrode structures limits the operating voltage and cathode field gradient in these guns. The photocathode operational lifetime is limited by the gun vacuum and by ion back bombardment. Recent developments in areas as diverse as vacuum technology, CW lasers with RF time structure, and the reduction of field emission from large area electrodes show promise for the development of a new generation of DC photoemission electron guns, operating at very high voltage and cathode field strengths well above those obtainable in the past. These developments, and various designs for very high voltage photoemission electron guns, will be reviewed.
\end{abstract}

Charles K. Sinclair", Cornell University, Ithaca, NY 14853, USA

\section{INTRODUCTION}

The successful demonstration of beam energy recovery at the Jefferson Laboratory IRFEL [1], coupled with the excellent performance of superconducting RF cavities at high accelerating gradient [2], has led to a number of ideas and proposals for $\mathrm{CW}$ electron accelerators for electron cooling [3], the production of synchrotron radiation [4], and linac-ring versions of a future electronion collider [5]. These machines all require high average current $\mathrm{CW}$ electron sources, frequently with demanding specifications on transverse and longitudinal emittances.

DC electron guns with photoemission cathodes are a natural choice for such applications. While RF guns operating at very high accelerating gradients are the current choice for delivering high charge electron bunches at low duty factor, their maximum accelerating gradient in CW operation is significantly lower [6]. Recent developments in the reduction of field emission from large area electrodes offer the possibility of operating DC guns with cathode field gradients comparable to those practical in CW RF guns [7].

High average current operation requires high quantum efficiency photocathodes, which are notoriously sensitive to the vacuum environment. Compared with RF guns, DC guns offer distinct advantages for the production of excellent vacuum. In DC guns, there are few restrictions on vacuum chamber geometry or the location and size of ports, and many choices for wall materials. They operate at ambient temperature without cooling.

*Work supported by Cornell University \#cks26@cornell.edu
Electron-optically, they have no time varying fields, and easily incorporate transverse focusing at the cathode.

To date, DC photoemission guns have employed GaAs photocathodes, and are used primarily for the production of low average currents of polarized electrons. Operating voltages have been modest, typically about $100 \mathrm{kV}$. The Jefferson Lab IRFEL gun is a significant exception to these statements. This gun has operated at $\sim 320 \mathrm{kV}$, delivering $5 \mathrm{~mA}$ of average current at bunch repetition rates of $\sim 75 \mathrm{MHz}$ [8]. It is presently being upgraded to operate at $500 \mathrm{kV}$ and $10 \mathrm{~mA}$ average current.

High average current, high brightness $\mathrm{CW}$ electron injectors will require significant $\mathrm{R} \& \mathrm{D}$ before they are realized. The technology choice between high voltage DC photoemission guns, normal conducting RF guns, and superconducting RF guns is a matter of current debate. In the sections below, we present information on the current issues and state-of-the-art with DC guns, and indicate where future improvements might lead.

\section{FIELD EMISSION}

Field emission is the principal effect limiting the operating voltage and electrode field strength in DC electron guns. It is the source of undesirable phenomena, such as charging of ceramic insulators, localized melting in areas struck by field emitted electrons, and vacuum degradation from both heating and electron stimulated desorption (ESD). These problems can lead to voltage breakdown of the cathode-anode gap, electrode surface damage, and punch through of the ceramic insulator.

In a gun with a Pierce electrode to provide focusing, the peak field on the electrode is two to three times higher than the field on the cathode. Electrodes are generally made from stainless steel. At field strengths approaching $10 \mathrm{MV} / \mathrm{m}$ field emission currents typically become unacceptable, and conservative gun designs limit electrode fields to values below this level. The strong dependence of field emission on field strength means that one does not have to reduce the field too greatly.

Many researchers have empirically explored field emission and its reduction by various surface treatments. These studies have usually been made on small area samples with small gaps over relatively short periods of time, making it difficult to apply a "good" experimental result to the real conditions in a high voltage gun. A test chamber was constructed at Jefferson Lab to study large area electrodes with moderate gaps. The electrodes had a uniform field area of $116 \mathrm{~cm}^{2}$. Voltages to $125 \mathrm{kV}$ were applied to several $\mathrm{mm}$ gaps during eight hour tests. We demonstrated a dramatic reduction in field emission from samples coated with $\sim 500 \mathrm{~nm}$ of silicon dioxide. Two samples showed field emission below $1.5 \mathrm{pA} / \mathrm{cm}^{2}$ at 30 $\mathrm{MV} / \mathrm{m}$, and undetectable emission below $22 \mathrm{MV} / \mathrm{m}$ [7]. 


\section{CERAMIC INSULATORS FOR HIGH VOLTAGE HOLDOFF}

A ceramic insulator isolates the high voltage applied to the cathode electrode and its support structure. Field emission originating at the ceramic-metal-vacuum triple junction is a well-understood cause of internal surface flashover, and is controlled by reducing the field on the junction with electrostatic shields. The external ceramic surface is usually fluted to inhibit flashover.

Charging caused by small field emission currents is a serious problem due to the exceptionally high ceramic bulk resistivity. Various ways of overcoming this problem have been developed. An insulator comprised of a series of ceramic rings separated by appropriately shaped metal electrodes can prevent field-emitted electrons from striking the ceramic. The electrodes are joined with external resistors to grade the potential uniformly [9]. Coatings with suitable sheet resistance have been used. More recently, ion implantation has been shown to produce a stable uniform sheet resistance on the surface of a ceramic [10]. While difficult to adapt to a large ceramic, this is an otherwise attractive solution. A sheet resistance of 50 to $100 \mathrm{Gohm} / \mathrm{square}$ is appropriate. The ideal solution may be to develop a ceramic with a stable homogeneous bulk resistivity of about $70 \mathrm{Gohm}-$ $\mathrm{cm}$. The temperature dependence of these high resistivities must not allow thermal runaway. Finally, the "inverted" gun design, described below, avoids the problem of field-emitted electrons striking the ceramic.

\section{PHOTOCATHODE CHOICES}

For any laser illuminated linear photoemitter, the photocurrent is given in terms of the laser wavelength and power and the cathode quantum efficiency by:

$$
i(m A)=\frac{\lambda(n m)}{124} \bullet P_{\text {laser }}(W) \bullet Q . E .(\%)
$$

This expression gives the minimum product of laser power and quantum efficiency to produce the current, since it assumes every electron and laser photon is used. If, for example, photons are lost in shaping the laser beam temporally or spatially, a higher raw laser power is required.

There are three families of practical high quantum efficiency photoemitters. These are alkali antimonides, alkali tellurides, and III-V semiconductors. All are p-type semiconductors. The first two have positive electron affinity (PEA), while the III-V semiconductors have negative electron affinity (NEA). In the PEA case, the bottom of the conduction band in the bulk material lies below the vacuum level outside the cathode, while in the NEA case, the conduction band minimum lies above the external vacuum level. This difference has important consequences for the cathode thermal emittance. Table 1 gives the operating parameters of typical photocathodes from each of the three high quantum efficiency families.

Photoemission from these cathodes is described by the "three step" model. These steps are (a) photon absorption
Table 1. Typical Cathode Operating Characteristics

\begin{tabular}{|l|c|c|}
\hline $\begin{array}{l}\text { Typical } \\
\text { Cathode }\end{array}$ & $\begin{array}{l}\text { Operating } \\
\text { Wavelength } \\
(\mathrm{nm})\end{array}$ & $\begin{array}{l}\text { P X Q.E. to } \\
\text { produce a 1 A } \\
\text { current (Watt-\%) }\end{array}$ \\
\hline $\mathrm{K}_{2} \mathrm{CsSb}$ & 527 & 235 \\
\hline $\mathrm{KCsTe}$ & 266 & 466 \\
\hline GaAs $(\mathrm{Cs}, \mathrm{F})$ & 780 & 159 \\
\hline
\end{tabular}

in the bulk cathode material; (b) electron diffusion to the cathode surface; and (c) electron emission through the surface potential barrier. Since the semiconductors are ptype, their conduction band is empty. The incident light is absorbed by valence band electrons, which are promoted to the conduction band. Electrons in the conduction band experience electron-phonon collisions, in which they lose energy and change direction as they diffuse toward the cathode surface. This energy loss continues until the electron reaches the conduction band minimum, where it remains until it is either emitted or recombines.

In a PEA cathode, electrons at the conduction band minimum are energetically prevented from being emitted, unlike in the NEA case. Electrons excited to the conduction band in NEA materials are likely to thermalize at the band minimum before they are emitted if the exciting photon energy is not too great. The emitted electrons thus originate from a population with an effective temperature close to the physical temperature of the cathode, resulting in a very low thermal emittance. This is in direct contrast to PEA emitters. Several emittance measurements of the beams from NEA GaAs photoemitters have confirmed the low thermal emittance, down to temperatures of $77 \mathrm{~K}$ [11].

Optical absorption in the antimonides and tellurides is very much greater than in the III-V materials, leading them to have a much faster temporal response, since the electrons have a much shorter distance to diffuse to reach the cathode surface. III-V photocathodes can support electron pulses no shorter than about $20-40 \mathrm{ps}$, while the antimonide and telluride cathodes support ps or shorter duration pulses.

\section{LASER SYSTEMS}

Lasers with RF time structure are required to produce a beam bunched at the photocathode, for subsequent acceleration in an RF linac. The time structure is generally produced in an actively mode-locked laser. At high repetition rates, the laser cavity length becomes impractically short, and in this case harmonic modelocking may be used [12].

A convenient method of harmonic mode-locking was demonstrated at Jefferson Lab. Light from an RF gainswitched diode laser was injected into a Ti:sapphire laser cavity. The RF was derived from the accelerator master oscillator. This scheme has produced $>2 \mathrm{~W}$ average power pulse trains stably locked to the accelerator RF at rates between $\sim 200 \mathrm{MHz}$ and $3 \mathrm{GHz}$ [13]. The fundamental wavelength of Ti:sapphire lasers is well matched to GaAs photocathodes. Ti:sapphire lasers 
operating at much higher power levels have been developed, and a version of this system operating at $\sim 10$ $\mathrm{W}$ appears feasible [14].

Alkali antimonide and telluride photocathodes require shorter wavelength illumination, presently provided by frequency doubling or quadrupling Nd:YAG or Nd:YLF lasers, or frequency doubling or tripling Ti:sapphire lasers. Quantum efficiencies of $10 \%$ or greater have been prepared on cathodes of each type in Table 1, implying that average optical powers of $\sim 2-5 \mathrm{~W}$ are necessary to generate $100 \mathrm{~mA}$ average current. In practice, one needs optical power well above this minimum, to accommodate degradation of the quantum efficiency with beam delivery. This laser power requirement is far more challenging at shorter wavelengths, when the inefficiencies of frequency multiplication are included. The use of alkali telluride photocathodes at high average current appears particularly daunting in this regard.

\section{PHOTOCATHODE LIFETIME AND VACUUM ISSUES}

Nothing happens during photoemission that degrades the quantum efficiency of a photocathode. However, all high quantum yield photocathodes are chemically reactive, and are degraded by chemically active gas species in the vacuum. For example, exposures to a small fraction of a Langmuir of $\mathrm{H}_{2} \mathrm{O}$ or $\mathrm{CO}_{2}$ can significantly degrade quantum efficiency. Photocathodes may also be damaged by ion back bombardment, independent of the gas species forming the ion. Thus, for long photocathode operational lifetimes, both the partial pressures of reactive gas species and the absolute pressure must be very low.

If the photocathode lifetime is long with no high voltage applied to the gun, one can be confident that harmful residual gases are not present. Static photocathode lifetimes of thousands of hours have been demonstrated at many laboratories and with all three photocathode types. At Jefferson Lab, one GaAs cathode showed no measurable loss of quantum efficiency on a cathode exposed to the static vacuum for several months, corresponding to a $1 /$ e lifetime over 20,000 hours.

Field-emitted electrons may be a source of residual gases through ESD, and thus shorten the cathode life when high voltage is applied to the gun. While measurements of cathode lifetime with high voltage applied but no illumination have not been made for extended times, information on this point can be had by observing the lifetime at low average beam current. For most guns constructed to date, ESD from field-emitted electrons has not caused significant quantum efficiency degradation. This situation may prove different in DC guns operating at very high electric fields.

Electrons originating at the cathode and striking downstream vacuum chamber walls can generate a significant gas load. This was observed in the Jefferson Lab polarized guns, and was traced to electrons originating from very large radius on the cathode, near the junction between the cathode and the focusing (i.e.
Pierce) electrode. Electrons from this region receive a substantial transverse kick from the locally large transverse fields, and follow extreme trajectories that ultimately strike beam tube walls. Deadening the quantum efficiency at large radius cured this problem, and resulted in lifetime improvements of factors of a thousand or more [15].

When all reactive gases are eliminated, ion back bombardment remains as a source of photocathode degradation. This mechanism has a clear signature, since the photocathode typically has an active area much larger than the illuminated area. In such circumstances, and with focusing at the cathode, electrons originating from off-center on the cathode follow trajectories that move toward the electrostatic axis of the gun. The ions produced, however, are accelerated directly back to the cathode with very little transverse displacement. Thus, the ions damage only regions of the cathode radially inside the electron emission point, along a line joining the emission point and the electrostatic center of the cathode. This damage signature has been clearly observed in several DC guns. While the details of the ion damage mechanisms are not clear, the solution is to reduce the vacuum pressure.

When ion back bombardment is the cathode lifetime limiting phenomenon, the cathode life is better expressed in terms of the charge delivered per unit illuminated area, rather than in clock hours or charge delivered. The best GaAs cathode lifetime reported to date is a $1 / \mathrm{e}$ degradation of the quantum efficiency from the delivery

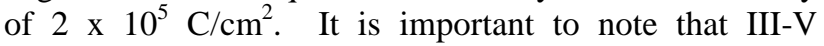
cathodes are bulk materials with a monatomic surface dipole layer, while the alkali antimonide and telluride cathodes are stoichiometric compounds. Thus, the response of these cathodes to ion back bombardment may be quite different.

In a DC gun with no ESD gas load, outgassing is the source of residual gas. This can be greatly reduced by the use of non-evaporable getter coatings sputtered on the chamber walls [16]. These coatings have very low outgassing, very high pumping speed for chemically active gases, very low ESD, and act as a diffusion barrier to gases permeating through the chamber walls.

\section{LOAD LOCKS AND GUN DESIGNS}

No high quantum efficiency cathode can be transferred through air without being destroyed, and it seems unlikely that a suitably inert atmospheric pressure environment can be established to allow such transfers without degradation. Attempts to prepare protective coatings on photocathodes have been made, but to date all have caused a loss in the quantum efficiency to levels unsuited for high average current use [17]. Thus, it is necessary to either prepare cathodes in situ in the electron guns, or transfer them under vacuum from a separate preparation chamber, using a load lock.

Three reasons are generally given in support of using a load lock system. These are that with a load lock (a) it is 
possible to have multiple cathodes prepared, ready for transfer into the gun; (b) the gun vacuum, once established, is never disturbed by cathode exchanges; and (c) there is no risk of contamination of high field electrode surfaces with alkali metals.

The first two of these reasons are weakened somewhat by operating experience with the Jefferson Lab polarized sources. Very long cathode lifetimes, ease of repeatedly cleaning and re-activating cathodes to their original quantum efficiency, and demonstrably excellent vacuum conditions were all reliably obtained in guns with no load lock. However, contamination of high field strength electrode surfaces with even tiny amounts of alkali metals could easily lead to unacceptably high levels of field emission, particularly in guns designed to operate at high field strength, and is reason enough to use a load lock. No doubt all future very high voltage DC photoemission guns will employ load locks.

The simplest gun design involves no load lock. An example is shown in figure 1. This gun, designed to operate at $500 \mathrm{kV}$ for the Jefferson Lab IRFEL, has a GaAs wafer mounted on a long cathode "stalk". Installation of the cathode stalk involves breaking the gun vacuum. Following a vacuum bakeout after cathode installation, the cathode is activated by the introduction of cesium through the anode aperture. In practice, though the gun could be processed to full voltage before the

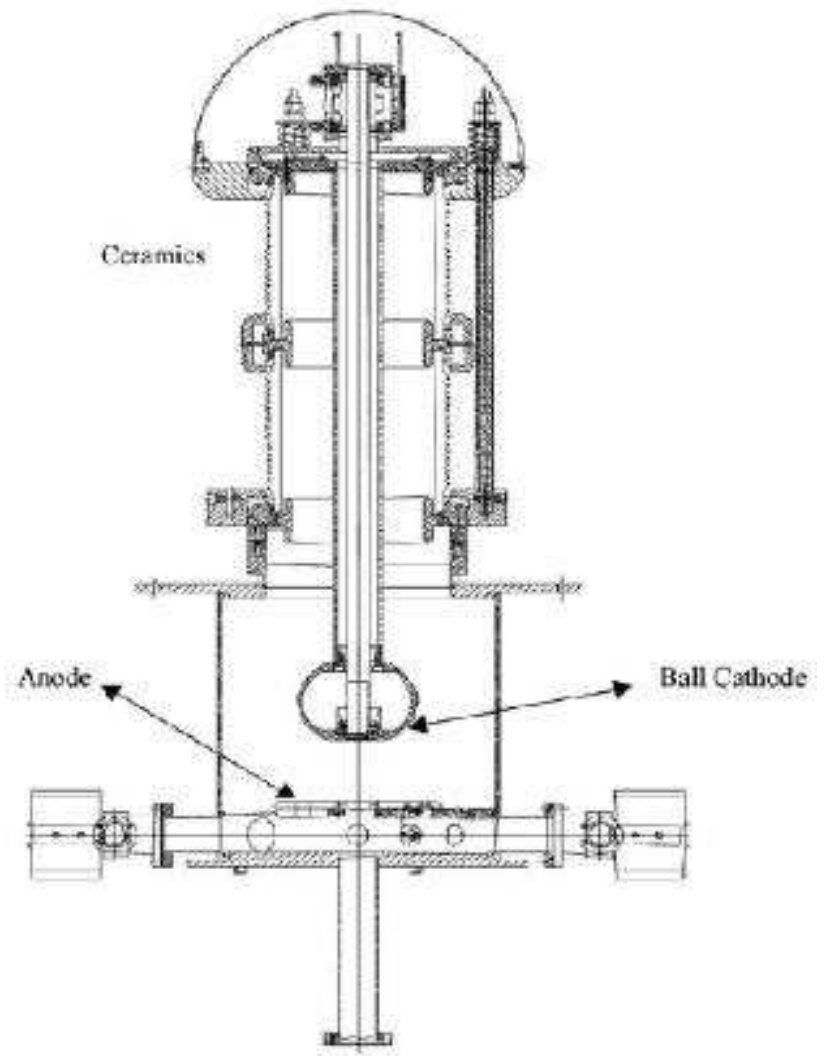

Figure 1. The $500 \mathrm{kV}$ gun at the JLab IRFEL

cathode was activated, after activation field emission was excessive above $320-335 \mathrm{kV}$. The gun did operate reliably for extended periods of time and multiple cathode activations at this reduced voltage [8].
As there is significant space available within the large cathode electrode, this gun has been redesigned to allow the cathode to be activated while retracted completely within this electrode, greatly reducing the likelihood of cesium contamination on the high field surfaces. A shutter blocks the aperture in the cathode electrode during high voltage processing. These changes are expected to allow the gun to reach its $500 \mathrm{kV}$ design voltage, and will soon be tested.

The simplest implementation of a load lock places the entire cathode preparation chamber and load lock transfer mechanism at cathode potential. This solution was adopted for the SLC polarized source, which operated at $120 \mathrm{kV}$ [18]. It becomes increasingly awkward to implement as the operating voltage is increased. In a design developed at Mainz, the cathode was moved into the cathode-anode gap, allowing cathode preparation and transfer mechanisms to be mounted at ground potential [19]. There are concerns that the edges of the cathode electrode could become a source of field emission in a very high voltage gun of this design.

Another way to locate the cathode preparation and transfer mechanism at ground potential is to use a second ceramic insulator between cathode potential and ground. Cathode transfer takes place along the axis of this second insulator. This solution was developed by Novosibirsk for the $100 \mathrm{kV}$ NIKHEF polarized source [20], and has been adopted for a $200 \mathrm{kV}$ gun built at Nagoya [21]. At high voltages, the transfer distance can become quite long.

A clever scheme known as the "inverted" gun was developed at SLAC, and shown in figure 2 [22]. The cathode electrode is supported within the gun chamber on ceramic rods, with the cathode preparation and transfer mechanisms at ground. High voltage is brought in through the bore of one of the rods, but could also be delivered through a separate feedthrough. In this design, field emitted electrons cannot reach the ceramic rods. The

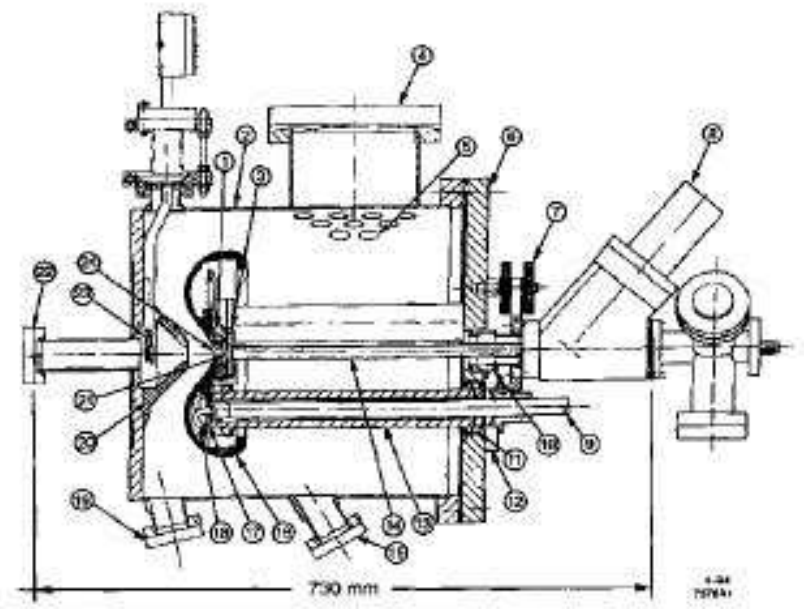

Figure 2. The inverted gun developed at SLAC

SLAC gun was designed to reach $200 \mathrm{kV}$, and operated there, though with very high levels of field emission, which were not understood. 
Another load lock gun design has been developed at Jefferson Lab. The cathode electrode is a hollow cylinder, isolated by a conventional ceramic, as shown in figure 3 [23]. The cathode is introduced through one end of this cylinder and secured into its operating location at the other end. This design incorporated an atomic hydrogen cleaning chamber, allowing a cathode sample to be cleaned prior to being moved into the preparation chamber. With this system, a cathode sample has been taken from the laboratory environment to beam delivery in several hours.

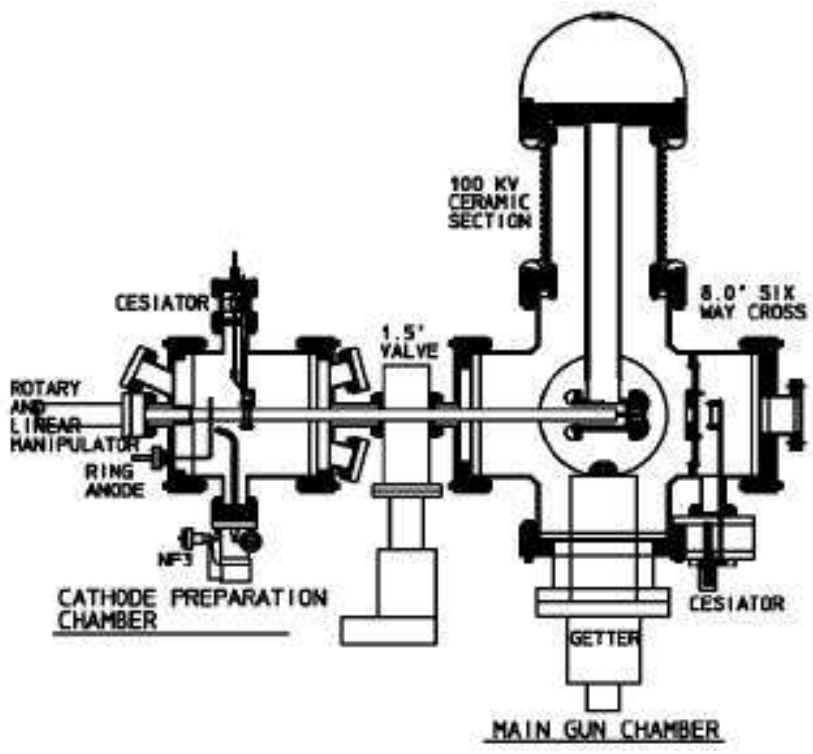

Figure 3. The JLab load-locked gun design

\section{CONCLUSIONS}

Very high voltage photoemission electron guns may well be able to meet the requirements for a number of currently envisioned CW electron accelerators. Methods for dramatically reducing the field emission from large area electrodes set the stage for operating such guns with electrode field strengths of $20 \mathrm{MV} / \mathrm{m}$ or more. Ion implantation, the development of ceramics with appropriate bulk restivities, insulator designs incorporating internal protective rings, or the use of the inverted gun design can eliminate the problem of ceramic charging from even small field emission currents. Various load-locked gun designs operating reliably and breakdown free at voltages above $500 \mathrm{kV}$ and at cathode field strengths above $10 \mathrm{MV} / \mathrm{m}$ now seem within reach.

For high average current operation, only high quantum efficiency photocathodes can be considered. Of the three families of such photocathodes, the alkali antimonides and tellurides have been used in RF guns, while NEA GaAs cathodes have been used in DC guns. In the GaAs cathode/DC gun case, the cathode operational lifetime is limited by ion back bombardment. The best $1 / \mathrm{e}$ lifetime reported to date is $2 \times 10^{5} \mathrm{C} / \mathrm{cm}^{2}$. The lifetime of the alkali antimonide and telluride cathodes may well be better than GaAs in a DC gun. The ion back bombardment lifetime may be improved by reducing the total vacuum pressure, which appears practical using NEG coatings in the gun vacuum chamber.

Lasers with the necessary RF time structure pose a challenge for high average current operation. The GaAs cathode offers an advantage, as the fundamental wavelength of a Ti:sapphire laser is suitable. Alkali antimonide cathodes require frequency doubled $\mathrm{Nd}$ or $\mathrm{Ti}$ lasers, while the tellurides require frequency quadrupled $\mathrm{Nd}$, or frequency tripled $\mathrm{Ti}$ lasers, and thus require more fundamental laser power to overcome the inefficiency of frequency multiplication. Fundamental frequency or harmonic mode-locked $\mathrm{Nd}$ or Ti lasers delivering $10 \mathrm{~W}$ of optical power with suitable RF time structure are well within reach.

NEA GaAs offers a significantly lower thermal emittance per unit illuminated area, compared to PEA cathodes, while PEA cathodes offer far shorter pulse durations. In a DC gun followed by a buncher, the long pulse from NEA GaAs may not be a significant drawback. Based on all these considerations, we plan to construct a 500 to $750 \mathrm{kV}$ gun with a NEA GaAs cathode to provide a $1300 \mathrm{MHz}$ train of $77 \mathrm{pC}$ bunches - 100 ma average current - for the Cornell/JLab ERL prototype [4].

\section{REFERENCES}

[1] G. R. Neil et al., Phys. Rev. Letters $\underline{\mathbf{8 4}}, 662$ (2000).

[2] B. Aune et al., Phys. Rev. ST-AB $\underline{\mathbf{3}}, 092001$ (2000).

[3] V. Parkhomchuk and I. Ben-Zvi, ref. 47 at http://www.agsrhichome.bnl.gov/ap_notes/cad_ap_in dex.html

[4] CHESS Technical Memo 01-003, available at http://erl.chess.cornell.edu/papers/ERL_Study.pdf

[5] L. Merminga et al., EPAC 2002, p. 203

[6] R. A. Rimmer et al., EPAC 2002, p. 1801

[7] C. K. Sinclair et al., PAC 2001, p. 610.

[8] T. Siggins et al., NIM A $\underline{\mathbf{4 7 5}}, 549$ (2001)

[9] J. Haimson, IEEE Trans. Nucl. Sci. NS-22, 1354 (1975)

[10] S. Anders et al., PAC 1993, p. 1390

[11] B. M. Dunham et al., PAC 1995, p. 1030; D. A. Orlov et al., Appl. Phys. Letters 78, 2721 (2001)

[12] M. F. Becker et al., IEEE J. QE, $\underline{\mathbf{8}}, 687$ (1972).

[13] C. Hovater and M. Poelker, NIM A 418, 280 (1998)

[14] W. J. Wadsworth et al., Appl. Opt. 38, 6904 (1999)

[15] C. K. Sinclair, PAC 1999, p. 65; K. Aulenbacher, private communication.

[16] C. Benvenuti et al., JVST A 16, 148 (1998), Y. Li and S. Ho, these proceedings.

[17] A. Breskin et al., Appl. Phys. Letters 69, 1008 (1996).

[18] R. Alley et al., NIM A $\underline{\mathbf{3 6 5}}, 1$ (1995).

[19] K. Aulenbacher et al., NIM A $\underline{\text { 391, }} 498$ (1997).

[20] C. W. de Jager et al., AIP Conf. Proc. No. 421, p. 483 (1997).

[21] M. Yamamoto et al., LINAC 2002, to be published.

[22] M. Breidenbach et al., NIM A $\underline{350}, 1$ (1994).

[23] W. J. Schneider et al., PAC 1999, p. 1991 